Commun.Fac.Sci.Univ.Ank.Series A 1

Volume 58, Number 2, Pages 39-46 (2009)

ISSN $1303-5991$

\title{
NOTES ON COMMUTATIVITY OF PRIME RINGS WITH GENERALIZED DERIVATION
}

\author{
ÖZNUR GÖLBAŞI AND EMINE KOÇ
}

\begin{abstract}
In this paper, we extend the results concerning generalized derivations of prime rings in [2] and [8] for a nonzero Lie ideal of a prime ring $R$.
\end{abstract}

\section{INTRODUCTION}

Let $R$ denote an associative ring with center $Z$. For any $x, y \in R$, the symbol $[x, y]$ stands for the commutator $x y-y x$. Recall that a ring $R$ is prime if $x R y=0$ implies $x=0$ or $y=0$. An additive mapping $d: R \rightarrow R$ is called a derivation if $d(x y)=d(x) y+x d(y)$ holds for all $x, y \in R$.

Recently, M. Bresar defined the following notation in [6]. An additive mapping $f: R \rightarrow R$ is called a generalized derivation if there exists a derivation $d: R \rightarrow R$ such that

$$
f(x y)=f(x) y+x d(y), \text { for all } x, y \in R .
$$

One may observe that the concept of generalized derivation includes the concept of derivations, also of the left multipliers when $d=0$. Hence it should be interesting to extend some results concerning these notions to generalized derivations.

Let $S$ be a nonempty subset of $R$. A mapping $f$ from $R$ to $R$ is called centralizing on $S$ if $[f(x), x] \in Z$ for all $x \in S$ and is called commuting on $S$ if $[f(x), x]=0$ for all $x \in S$. The study of such mappings was initiated by E. C. Posner in [12]. During the past few decades, there has been an ongoing interest concerning the relationship between the commutativity of a ring and the existence of certain specific types of derivations of $R$. In [4], R. Awtar proved that a nontrivial derivation which is centralizing on Lie ideal implies that the ideal is contained in the center a prime ring $R$ with characteristic different from two or three. P. H. Lee and T. K. Lee obtained same result while removing the characteristic not three restriction in [11]. In [3], N. Argaç and E. Albaş extended this result for generalized derivations of a prime ring $R$ and in [8], Ö. Gölbaşı proved the same result for a semiprime ring $R$.

Received by the editors Feb. 23, 2009; Accepted: Dec. 12, 2009.

1991 Mathematics Subject Classification. 16W25, 16N60, 16 U80.

Key words and phrases. prime rings, derivations, generalized derivations, lie ideals. 
The first purpose of this paper is to show this theorem for a nonzero Lie ideal $U$ of $R$ such that $u^{2} \in U$ for all $u \in U$.

On the other hand, in [1], M. Asraf and N. Rehman showed that a prime ring $R$ with a nonzero ideal $I$ must be commutative if it admits a derivation $d$ satisfying either of the properties $d(x y)+x y \in Z$ or $d(x y)-x y \in Z$, for all $x, y \in R$. In [2], the authors explored the commutativity of prime $\operatorname{ring} R$ in which satisfies any one of the properties when $f$ is a generalized derivation:

(i) $f(x y)-x y \in Z$,

(ii) $f(x y)+x y \in Z,($ iii $) f(x y)-y x \in Z$,

(iv) $f(x y)+y x \in Z(v) f(x) f(y)-x y \in Z$

$(v i) f(x) f(y)+x y \in Z$,

for all $x, y \in R$. The second aim of this paper is to prove these theorems for a nonzero Lie ideal $U$ of $R$ such that $u^{2} \in U$ for all $u \in U$.

\section{Preliminaries}

Throughout the paper, we denote a generalized derivation $f: R \rightarrow R$ determined by a derivation $d$ of $R$ with $(f, d)$ and make some extensive use of the basic commutator identities:

$[x, y z]=y[x, z]+[x, y] z$

$[x y, z]=[x, z] y+x[y, z]$

Notice that $u v+v u=(u+v)^{2}-u^{2}-v^{2}$ for all $u, v \in U$. Since $u^{2} \in U$ for all $u \in U, u v+v u \in U$. Also $u v-v u \in U$, for all $u, v \in U$. Hence, we find $2 u v \in U$ for all $u, v \in U$.

Moreover, we shall require the following lemmas.

Lemma 2.1. [9, Lemma 1] Let $R$ be a semiprime, 2-torsion free ring and $U$ a nonzero Lie ideal of $R$. Suppose that $[U, U] \subset Z$, then $U \subseteq Z$.

Definition 2.2. Let $R$ be a ring, $A \subset R . C(A)=\{x \in R \mid x a=a x$, for all $a \in A\}$ is called the centralizer of $A$.

Lemma 2.3. [5, Lemma 2] Let $R$ be a prime ring with characteristic not two. If $U$ a noncentral Lie ideal of $R$, then $C_{R}(U)=Z$.

Lemma 2.4. [5, Lemma 4] Let $R$ be a prime ring with characteristic not two, $a, b \in R$. If $U$ a noncentral Lie ideal of $R$ and $a U b=0$, then $a=0$ or $b=0$.

Lemma 2.5. [5, Lemma 5] Let $R$ be a prime ring with characteristic not two and $U$ a nonzero Lie ideal of $R$. If $d$ is a nonzero derivation of $R$ such that $d(U)=0$, then $U \subseteq Z$.

Lemma 2.6. [5, Theorem 2] Let $R$ be a prime ring with characteristic not two and $U$ a noncentral Lie ideal of $R$. If $d$ is a nonzero derivation of $R$, then $C_{R}(d(U))=Z$.

Lemma 2.7. [11, Theorem 5] Let $R$ be a prime ring with characteristic not two and $U$ a nonzero Lie ideal of $R$. If $d$ is a nonzero derivation of $R$ such that $[u, d(u)] \in Z$, for all $u \in U$, then $U \subseteq Z$. 


\section{Results}

The following theorem gives a generalization of Posner's well known result [12, Lemma 3] and a partial extension of [7, Theorem 4.1].

Theorem 3.1. Let $R$ be a 2 -torsion free prime ring and $U$ a nonzero Lie ideal of $R$ such that $u^{2} \in U$ for all $u \in U$. If $R$ admits nonzero generalized derivations $(f, d)$ and $(g, h)$ such that $f(u) v=u g(v)$, for all $u, v \in U$, and if $d, h \neq 0$, then $U \subseteq Z$.

Proof. We have

$$
f(u) v=u g(v), \text { for all } u, v \in U .
$$

Replacing $u$ by $[x, u] u, x \in R$ in (3.1) and applying (3.1), we get

$$
\begin{aligned}
& f([x, u]) u v+[x, u] d(u) v=[x, u] u g(v) \\
& {[x, u] g(u) v+[x, u] d(u) v=[x, u] u g(v),}
\end{aligned}
$$

and so

$$
[x, u](g(u) v+d(u) v-u g(v))=0, \text { for all } u, v \in U, x \in R .
$$

Substituting $x y$ for $x$ in (3.2) and using this, we get

$$
[x, u] R(g(u) v+d(u) v-u g(v))=0, \text { for all } u, v \in U, x \in R .
$$

Since $R$ is prime ring, the above relation yields that

$$
u \in Z \text { or } g(u) v+d(u) v-u g(v)=0, \text { for all } v \in U, x \in R .
$$

We set $K=\{u \in U \mid u \in Z\}$ and $L=\{u \in U \mid g(u) v+d(u) v-u g(v)=0$, for all $v \in U\}$. Clearly each of $K$ and $L$ is additive subgroup of $U$. Morever, $U$ is the set-theoretic union of $K$ and $L$. But a group can not be the set-theoretic union of two proper subgroups, hence $K=U$ or $L=U$.

In the latter case, $g(u) v+d(u) v-u g(v)=0$, for all $u, v \in U$. Now, taking $2 v w$ instead of $v$ in this equation and using this, we have

$$
u v h(w)=0, \text { for all } u, v, w \in U .
$$

That is $u U h(U)=(0)$, for all $u \in U$. By Lemma 2.4 and Lemma 2.5, we get $u=0$ or $U \subseteq Z$. This implies $U \subseteq Z$ for any cases.

Corollary 1. Let $R$ be a 2 -torsion free prime ring and $U$ a nonzero Lie ideal of $R$ such that $u^{2} \in U$ for all $u \in U$. If $R$ admits nonzero generalized derivations $(f, d)$ and $(g, h)$ such that $f(u) u=u g(u)$, for all $u \in U$, and if $d, h \neq 0$, then $U \subseteq Z$.

Corollary 2. Let $R$ be a 2 -torsion free prime ring and $U$ a nonzero Lie ideal of $R$ such that $u^{2} \in U$ for all $u \in U$. If $R$ admits a nonzero generalized derivation $(f, d)$ such that $[f(u), u]=0$, for all $u \in U$, and if $d \neq 0$, then $U \subseteq Z$.

Corollary 3. Let $R$ be a 2 -torsion free prime ring. If $R$ admits nonzero generalized derivations $(f, d)$ and $(g, h)$ such that $f(x) y=x g(y)$, for all $x, y \in R$, and if $d, h \neq 0$, then $R$ is commutative ring. 
Corollary 4. Let $R$ be a 2 -torsion free prime ring. If $R$ admits nonzero generalized derivations $(f, d)$ and $(g, h)$ such that $f(x) x=x g(x)$, for all $x \in R$, and if $d, h \neq 0$, then $R$ is commutative ring.

Using the same techniques with necessary variations in the proof of Theorem 3.1 , we can give the following corollary which a partial extends [3, Lemma 12] even without the characteristic assumption on the ring.

Corollary 5. Let $R$ be prime ring concerning a nonzero generalized derivation $(f, d)$ such that $[f(x), x]=0$, for all $x \in R$, and if $d \neq 0$, then $R$ is commutative ring.

Lemma 3.2. Let $R$ be a prime ring with characteristic not two, $a \in R$. If $U$ a noncentral Lie ideal of $R$ such that $u^{2} \in U$ for all $u \in U$ and $a U \subseteq Z(U a \subseteq Z)$ then $a \in Z$.

Proof. By the hyphotesis, we have

$$
[a u, a]=0,
$$

and so

$$
a[u, a]=0, \text { for all } u \in U .
$$

Replacing $u$ by $2 u v$ in this equation, we arrive at

$$
a u[v, a]=0, \text { for all } u, v \in U .
$$

We get $a=0$ or $[v, a]=0$, for all $v \in U$, by Lemma 2.4 , and so $a \in Z$ by Lemma 2.3 .

Theorem 3.3. Let $R$ be a 2 -torsion free prime ring and $U$ a nonzero Lie ideal of $R$ such that $u^{2} \in U$ for all $u \in U$. If $R$ admits a generalized derivation $(f, d)$ such that $f(u v)-u v \in Z$, for all $u, v \in U$, and if $d \neq 0$, then $U \subseteq Z$.

Proof. If $f=0$, then $u v \in Z$ for all $u, v \in U$. In particular $u U \subseteq Z$, for all $u \in U$. Hence $U \subseteq Z$ by Lemma 3.2. Hence onward we assume that $f \neq 0$.

By the hyphotesis, we have

$$
f(u) v+u d(v)-u v \in Z, \text { for all } u, v \in U .
$$

Replacing $u$ by $2 u w$ in (3.3), we get

$$
2((f(u w)-u w) v+u w d(v)) \in Z \text {, for all } u, v, w \in U .
$$

Commuting this term with $v \in U$, we arrive at

$$
u w[d(v), v]+u[w, v] d(v)+[u, v] w d(v)=0, \text { for all } u, v, w \in U .
$$

Taking $u$ by $2 t u$ in (3.4) and using this equation, we get

$$
[t, v] u w d(v)=0 \text {, for all } u, v, w, t \in U .
$$

We can write $[t, v] U d(v)=0$, for all $v, t \in U$. This yields that

$$
[t, v]=0 \text { or } d(v)=0 \text {, for all } t \in U \text {. }
$$


by Lemma 2.4. We set

$$
K=\{v \in U \mid[t, v]=0, \text { for all } t \in U\}
$$

and

$$
L=\{v \in U \mid d(v)=0\} .
$$

Then by Braur's trick, we get either $U=K$ or $U=L$. In the first case, $U \subseteq Z$ by Lemma 2.3, and in the second case $U \subseteq Z$ by Lemma 2.5. This completes the proof.

Corollary 6. Let $R$ be a 2-torsion free prime ring. If $R$ admits a generalized derivation $(f, d)$ such that $f(x y)-x y \in Z$, for all $x, y \in R$, and if $d \neq 0$, then $R$ is commutative ring.

Theorem 3.4. Let $R$ be a 2 -torsion free prime ring and $U$ a nonzero Lie ideal of $R$ such that $u^{2} \in U$ for all $u \in U$. If $R$ admits a generalized derivation $(f, d)$ such that $f(u v)+u v \in Z$, for all $u, v \in U$, and if $d \neq 0$, then $U \subseteq Z$.

Proof. If $f$ is a generalized derivation satisfying the property $f(u v)+u v \in Z$, for all $u, v \in U$, then $(-f)$ satisfies the condition $(-f)(u v)-u v \in Z$, for all $u, v \in U$ and hence by Theorem $3.3, U \subseteq Z$.

Corollary 7. Let $R$ be a 2-torsion free prime ring. If $R$ admits a generalized derivation $(f, d)$ such that $f(x y)+x y \in Z$, for all $x, y \in R$, and if $d \neq 0$, then $R$ is commutative ring.

Theorem 3.5. Let $R$ be a 2 -torsion free prime ring and $U$ a nonzero Lie ideal of $R$ such that $u^{2} \in U$ for all $u \in U$. If $R$ admits a generalized derivation $(f, d)$ such that $f(u v)-v u \in Z$, for all $u, v \in U$, and if $d \neq 0$, then $U \subseteq Z$.

Proof. If $f=0$, then $v u \in Z$ for all $u, v \in U$. Applying the same arguments as used in the begining of the proof of Theorem 3.1, we get the required result. Hence onward we assume that $f \neq 0$.

By the hypothesis, we have

$$
f(u v)-v u \in Z \text {, for all } u, v \in U .
$$

Replacing $v$ by $2 w v$ in (3.5), we get $f(2 u w v)-2 w v u \in Z$, for all $u, v, w \in U$. Commuting this term with $v \in U$, we have

$$
[f(u w) v+u w d(v)-w v u, v]=0
$$

and so

$$
[f(u w) v-w u v+w u v+u w d(v)-w v u, v]=0, \text { for all } u, v, w \in U .
$$

Using the (3.5), we arrive at

$$
[w u v+u w d(v)-w v u, v]=0
$$

and so

$$
[w, v][u, v]+w[[u, v], v]+u w[d(v), v]+[u, v] w d(v)+u[w, v] d(v)=0 .
$$


Substituting $2 u w$ for $w$ in (3.6) equation and using this, we obtain that

$$
[u, v] w[u, v]+[u, v] u w d(v)=0, \text { for all } u, v, w \in U
$$

Now taking $v$ by $u+v$ in (3.7) and using this equation, we get

$$
[u, v] u w d(v)=0, \text { for all } u, v, w \in U .
$$

By Lemma 2.4, we get $[u, v] u=0$ or $d(v)=0$, for all $u \in U$. We set

$$
K=\{v \in U \mid[u, v] u=0, \text { forallu } \in U\}
$$

and

$$
L=\{v \in U \mid d(v)=0\}
$$

Then by Braur's trick, we get either $U=K$ or $U=L$. If $U=L$, then $U \subseteq Z$ by Lemma 2.5. If $U=K$, then $[u, v] u=0$, for all $u \in U$. Writing $v$ by $2 v t$ in this, we arrive at

$$
[u, v] t u=0, \text { for all } u, v, t \in U .
$$

Again using Lemma 2.4, we have $[u, v]=0$, for all $u, v \in U$, and so $U \subseteq Z$ by Lemma 2.3.

Corollary 8. Let $R$ be a 2-torsion free prime ring. If $R$ admits a generalized derivation $(f, d)$ such that $f(x y)-y x \in Z$, for all $x, y \in R$, and if $d \neq 0$, then $R$ is commutative ring.

Using similar arguments as above, we can prove the followings:

Theorem 3.6. Let $R$ be a 2 -torsion free prime ring and $U$ a nonzero Lie ideal of $R$ such that $u^{2} \in U$ for all $u \in U$. If $R$ admits a generalized derivation $(f, d)$ such that $f(u v)+v u \in Z$, for all $u, v \in U$, and if $d \neq 0$, then $U \subseteq Z$.

Corollary 9. Let $R$ be a 2-torsion free prime ring. If $R$ admits a generalized derivation $(f, d)$ such that $f(x y)+y x \in Z$, for all $x, y \in R$, and if $d \neq 0$, then $R$ is commutative ring.

Theorem 3.7. Let $R$ be a 2 -torsion free prime ring and $U$ a nonzero Lie ideal of $R$ such that $u^{2} \in U$ for all $u \in U$. If $R$ admits a generalized derivation $(f, d)$ such that $f(u) f(v)-u v \in Z$, for all $u, v \in U$, and if $d \neq 0$, then $U \subseteq Z$.

Proof. If $f=0$, then $u v \in Z$ for all $u, v \in U$. Applying the same arguments as used in the begining of the proof of Theorem 3.1, we get the required result. Hence onward we assume that $f \neq 0$.

By the hypothesis, we have $f(u) f(v)-u v \in Z$, for all $u, v \in U$. Writing $2 v w$ by $v$ in this equation yields that

$$
2((f(u) f(v)-u v) w+f(u) v d(w)) \in Z \text {, for all } u, v, w \in U .
$$

Commuting (3.8) with $w \in U$, we have

$$
[f(u) v d(w), w]=0, \text { for all } u, v, w \in U .
$$


Substituting $2 u t, t \in U$ for $u$ in (3.9), we obtain that

$$
2[f(u) \operatorname{tvd}(w), w]+2[u d(t) v d(w), w]=0,
$$

Using (3.9) in this equation, we get

$$
[u d(t) v d(w), w]=0, \text { for all } u, v, w, t \in U .
$$

That is

$$
u d(t)[v d(w), w]+[u d(t), w] v d(w)=0, \text { for all } u, v, w, t \in U .
$$

Replacing $v$ by $2 k d(m) v, k \in U, m \in[U, U]$ in this equation and using (3.10), we arrive at

$$
[u d(t), w] k d(m) v d(w)=0, \text { for all } u, v, w, t, k \in U, m \in[U, U] .
$$

By Lemma 2.4, we get either $[u d(t), w]=0$ or $d(m)=0$ or $d(w)=0$ for all $u, v, w, t, k \in U, m \in[U, U]$. If $d(m)=0$, for all $m \in[U, U]$, then $[U, U] \subset Z$ by Lemma 2.5, and so again using Lemma 2.1, we get $U \subseteq Z$. This completes the proof.

Now we assume either $[u d(t), w]=0$ or $d(w)=0$ for each $w \in U$. We set $K=\{w \in U \mid[u d(t), w]=0$, for all $u, t \in U\}$ and $L=\{w \in U \mid d(w)=0\}$. Clearly each of $K$ and $L$ is additive subgroup of $U$. Then by Braur's trick, we get either $U=K$ or $U=L$. In the second case, $U \subseteq Z$ by Lemma 2.5 .

In the first case, $[u d(t), w]=0$, for all $u, w, t \in U$. Replacing $w$ by $d(t), t \in[U, U]$ in this equation and using this, we arrive at

$$
[u, d(t)] d(t)=0, \text { for all } u \in U, t \in[U, U]
$$

Substituting $2 t u, u \in U$ for $u$ in (3.9) and using this, we obtain that

$$
[t, d(t)] u d(t)=0 \text {, for all } u \in U, t \in[U, U] .
$$

Let

and

$$
K=\{t \in[U, U] \mid[t, d(t)]=0\}
$$

$$
L=\{t \in[U, U] \mid d(t)=0\}
$$

of additive subgroups of $[U, U]$. Now using the same argument as we have done, we get $[U, U]=K$ or $[U, U]=L$. If $[U, U]=L$ then we have required result applying similar arguments as above. If $[U, U]=K$, then $[U, U] \subset Z$ by Lemma 2.7, and so again using Lemma 2.1, we get $U \subseteq Z$.

Corollary 10. Let $R$ be a 2 -torsion free prime ring. If $R$ admits a generalized derivation $(f, d)$ such that $f(x) f(y)-x y \in Z$, for all $x, y \in R$, and if $d \neq 0$, then $R$ is commutative ring.

Application of similar arguments yields the following.

Theorem 3.8. Let $R$ be a 2-torsion free prime ring and $U$ a nonzero Lie ideal of $R$ such that $u^{2} \in U$ for all $u \in U$. If $R$ admits a generalized derivation $(f, d)$ such that $f(u) f(v)+u v \in Z$, for all $u, v \in U$, and if $d \neq 0$, then $U \subseteq Z$. 
Corollary 11. Let $R$ be a 2 -torsion free prime ring. If $R$ admits a generalized derivation $(f, d)$ such that $f(x) f(y)+x y \in Z$, for all $x, y \in R$, and if $d \neq 0$, then $R$ is commutative ring.

ÖZET: Bu çalışmada, [2] ve [8] makalelerinde genelleştirilmiş türevli asal halkalar için elde edilen sonuçlar, sıfırdan farklı bir Lie ideal için incelenmiştir.

\section{REFERENCES}

[1] Ashraf, M., Rehman, N. On derivations and commutativity in prime rings, East-West J. Math. 3(1), 2001, 87-91.

[2] Ashraf, M., Asma, A., Shakir, A. Some commutativiy theorems for rings with generalized derivations, Southeast Asain Bull. of Math. 31, 2007, 415-421.

[3] Argaç, N., Albaş, E. Generalized derivations of prime rings, Algebra Coll. 11(3), 2004, 399410.

[4] Awtar, R. Lie and Jordan structure in prime rings with derivations", Proc. Amer. Math. Soc., 41, 1973, 67-74.

[5] Bergen, J., Herstein, I. N., Kerr, J. W. Lie ideals and derivation of prime rings, Journal of Algebra, 71, 1981, 259-267.

[6] Bresar, M. On the distance of the composition of two derivations to the generalized derivations, Glasgow Math. J., 33, 1991, 89-93.

[7] Bresar, M. Centralizing mappings and derivations in prime ring, Journal of Algebra, 156, 1993, 385-394.

[8] Gölbaşı, Ö. On commutativity of semiprime rings with generalized derivations, (to appear Indiana Journal of Pure and Applied Math.)

[9] Herstein, I. N. On the Lie structure of an assosiative ring, Journal of Algebra, 14, 1970, 561-771.

[10] Herstein, I. N. A note on derivations, Canad. Math. Bull., 21(3), 1978, 369-370.

[11] Lee, P. H., Lee, T. K. Lie ideals of prime rings with derivations, Bull. Institute of Math. Academia Sinica, 11, 1983, 75-79.

[12] Posner, E. C. Derivations in prime rings, Proc Amer. Math. Soc., 8, 1957, 1093-1100.

[13] Rehman, N. On commutativity of rings with generalized derivations, Math. J. Okayama Univ. 44, 2002, 43-49.

Current address: Cumhuriyet University, Faculty of Arts and Science, Department of Mathematics, Sivas - TURKEY

E-mail address: ogolbasi@cumhuriyet.edu.tr; eminekoc@cumhuriyet.edu.tr

$U R L:$ http://www. cumhuriyet.edu.tr 